\title{
Brown tree snakes effectively removed from a large plot of land on Guam by perimeter trapping
}

\author{
Richard M. Engeman $^{\mathrm{a}, *}$, Daniel S. Vice ${ }^{\mathrm{b}}$, George Nelson ${ }^{\mathrm{b}}$, Ernest Muña ${ }^{\mathrm{b}}$ \\ ${ }^{a}$ USDA/APHIS, National Wildlife Research Center, 4101 Laporte Avenue, Fort Collins, CO 80521-2154, USA \\ ${ }^{\mathrm{b}}$ USDA/APHIS/WS, 1060 Route 16, Suite 103-C, Barrigada Heights, GU 96921, USA
}

\begin{abstract}
The brown tree snake introduction to Guam has had serious environmental, economic and social consequences. Trapping brown tree snakes in the vicinities of ports and other cargo staging facilities is central to a program implemented to deter its dispersal from Guam. Trapping forested plots on their perimeters has been an efficient and effective trap placement strategy for removing brown tree snakes from plots up to 8.4 ha. Here we examined whether this trap placement strategy was effective on a 17.8 ha plot, over twice the size of plot for which there was solid evidence of perimeter trapping's efficacy. We found that brown tree snakes were removed according to an exponential decay function. From 7 weeks on of trapping, snake captures had declined to low steady state levels that may best reflect population recruitment in the plot. After 22 weeks of trapping, both the plot interior and perimeter were trapped in a second phase designed to determine if the central portion of the plot contained reservoirs of brown tree snake populations. The second trapping phase lasted for 8 weeks and produced the same low, steady state capture rates as the final 16 weeks of the first phase that used only perimeter trapping. Only five snakes were captured in the plot interior in the second phase. We concluded that perimeter trapping removed brown tree snakes throughout the plot and the strategy could be applied to larger plots than demonstrated previously. Published by Elsevier Science Ltd.
\end{abstract}

\section{Introduction}

The brown tree snake (Boiga irregularis) on Guam is a severe example of the negative effects that an introduced predator can have on insular populations of native fauna (Savidge, 1987). The brown tree snake was brought to Guam accidentally through post World War II shipments from New Guinea (Rodda et al., 1992) and its populations have attained extraordinary densities (Rodda et al., 1992). They have decimated the native fauna. Currently, only three of the 12 species of forest birds survive in the wild, with one of those on the verge of elimination (National Research Council, 1997). Bat populations declined along with the bird populations. Guam populations of Mariana fruit bat (Pteropus mariannus), already impacted by

\footnotetext{
*Corresponding author. Tel.: + 1-970-266-6091; fax: + 1-970-2666059.

E-mail address: richard.m.engeman@usda.gov (R.M. Engeman).
}

hunting, were further decimated by snake predation. Two other bat species disappeared by the early 1970's due to unidentified causes (Wiles et al., 1995). Only one of the 12 native species of lizards on Guam occur in densities similar to nearby snake-free islands (Rodda and Fritts, 1992).

Guam has also suffered economic and social consequences of the brown tree snake introduction. Brown tree snakes prey on poultry and other small domesticated animals (Fritts and McCoid, 1991). They climb utility poles and wires, causing frequent power failures that result in millions of dollars of damaged equipment, loss of productivity, and repair cost (Fritts et al., 1987). Furthermore, the brown tree snake is mildly venomous and readily enters buildings where it is a health hazard to small children, who are less able to defend themselves and have been the subjects of lifethreatening snake bite incidents (Fritts et al., 1990).

The brown tree snake is well-suited for transport to, and establishment at, other locations. They are agile climbers that seek refuge from heat and light during 
daylight. Cargo, shipping containers, and transport vessels may offer ready daytime refugia. These snakes are opportunistic feeders that consume a highly varied diet (Savidge, 1987; Rodda et al., 1997; Linnell et al., 1997). These elements, coupled with Guam's position as a focal point for commercial and military shipments of cargo and passengers throughout the Pacific, present an acute and chronic likelihood for further dispersal of brown tree snake from Guam. Sightings have been documented on many Pacific islands, with an incipient population speculated to exist on Saipan in the Commonwealth of the Northern Mariana Islands (CNMI) (McCoid et al., 1994).

Three control methods (Engeman et al., 1998b) are employed to minimize brown tree snake dispersal: (1) detector dog searches of outbound cargo, and (2) trapping and (3) night-time spotlight searches of fence lines to create low population buffer areas in the vicinities of cargo staging areas. Habitat adjacent to port, cargo packing and cargo staging areas is largely comprised of remnant plots of forested vegetation, fragmented by commercial, industrial or military development. Trapping these forested plots around cargo staging areas is central to this integrated wildlife damage management program.

Capture records from the federal program aimed at controlling brown tree snakes indicated that trapping on the forest perimeter was an effective, labor-efficient means for snake removal from plots as large as 8.4 ha (Engeman et al., 1998c). In that same paper, an 18.2 ha plot showed large fluctuations in brown tree snake capture rates over time, rather than a consistent trend of diminishing capture rates that would indicate steady population removal. However, the snake capture rates never rebounded to more than about half of the initial capture rates, implying some level of sustained efficacy, and there was a large amount of variability in the trapping effort (number of traps) applied to that plot, allowing for varying levels of recruitment to the snake population.

Ensuing studies on plots ranging in size from 3.6 to 6.5 ha have confirmed that a perimeter trap placement strategy is highly effective for snake removal from forested plots in this size range (Engeman and Linnell, 1998). Perhaps the efficacy of this trapping strategy derives from the tendency of snakes encountering the forest edge to stay along its perimeter, thus resulting in a higher probability of snakes encountering traps on the forest perimeter (Engeman et al., 1998c).

An assumption of perimeter trapping is that the size of the area trapped can affect its efficacy. At some point, perimeter trapping is no longer effective at removing brown tree snakes from the plot, but this size limit has yet to be determined. In particular, we seek to demonstrate that perimeter trapping can effectively remove the snake population in a plot much lar- ger than 8.4 ha in a reasonable amount of time (a minimum of 4 months typically is used for operational control purposes). This information would serve to optimize the use of personnel and resources devoted to trapping, while maximizing the extent of trapping efforts.

\section{Methods}

The study was conducted in 1997 over a 7 month period in the Naval Magazine facility in south-central Guam. The Naval Magazine has a network of roads that produce well-defined plots of forested land in a variety of shapes and sizes. This presents a similar situation to that where trapping for snake control takes place: developed areas with the forest habitat fragmented into well-defined plots, such as around air and sea ports and other cargo staging facilities. We also chose to use the Naval Magazine because of the high security associated with this munitions storage facility, greatly decreasing the prospects of trap tampering during the study.

We selected a forested 17.8 ha plot, roughly trapezoidal in shape, in the Naval Magazine for study because it was of similar size to the 18.2 ha plot that provided inconclusive results in the study of Engeman et al. (1998c). Trapping was conducted in two phases. In Phase 1, removal trapping was applied to the entire perimeter of the plot using the operational standard of a $20 \mathrm{~m}$ spacing between traps (150 traps total). The traps were of a funnel design (i.e., minnow or crayfish traps), with one-way doors installed at the entrances (e.g., Linnell et al., 1998). A live mouse, protected in an interior cage, served as an attractant. Brown tree snake and nontarget animal captures were monitored and recorded weekly.

After captures had dropped to a low, steady state level ( $<0.01$ snakes/trap-night) for 4 months, Phase 2 of trapping was implemented. In this phase, removal trapping was maintained at the same intensity on the plot perimeter and an additional 48 traps were placed through the plot interior ( $>30 \mathrm{~m}$ from plot perimeter) to determine if snakes remained there in consequential numbers. To thoroughly sample the plot interior, traps were placed through the interior in two parallel lines bisected by a third line. Trapping in the interior, in addition to the perimeter, was conducted for another 8 weeks.

\section{Results}

Phase 1 of trapping lasted for 22 weeks, but the capture rate for brown tree snakes rapidly declined to low levels, dropping from 0.0533 to 0.0043 snakes/trapnight at 7 weeks, and remained in a low steady state, 
ranging only from 0.0018 to 0.0074 snakes/trap-night during the following 15 weeks of Phase 1 trapping.

We modeled the decline in captures as an exponential decay function. We used the first 9 weeks of capture data to insure that captures had reached the steady state level. The following model $\left(R^{2}=0.88\right.$, $p=0.0002$ ) resulted (Fig. 1):

captures $=0.092 \mathrm{e}^{-0.381 \text { week }}$.

The capture rates for the weeks in Phase 1 following the modeled data averaged only 0.0047 snakes/trapnight and, with $R^{2}=0.01$, showed no time trend up or down.

During Phase 2 ( 8 weeks beyond Phase 1), only five of the brown tree snakes were captured from interior traps, all in the first 3 weeks. The weekly capture rates for perimeter and interior traps combined remained at low levels similar to the capture rates for the final 16 weeks of Phase 1, ranging from 0.0010 to 0.0095 snakes/trap-night. This additional trapping averaged 0.0060 snakes/trap-night and continued to show no time trend up or down with an $R^{2}$ of only 0.18 with time (weeks). No differences in weekly brown tree snake capture rates were detected between Phase 2 and the period following the initial decline (first 6 weeks) in capture rates in Phase 1 (Wilcoxon rank sum,

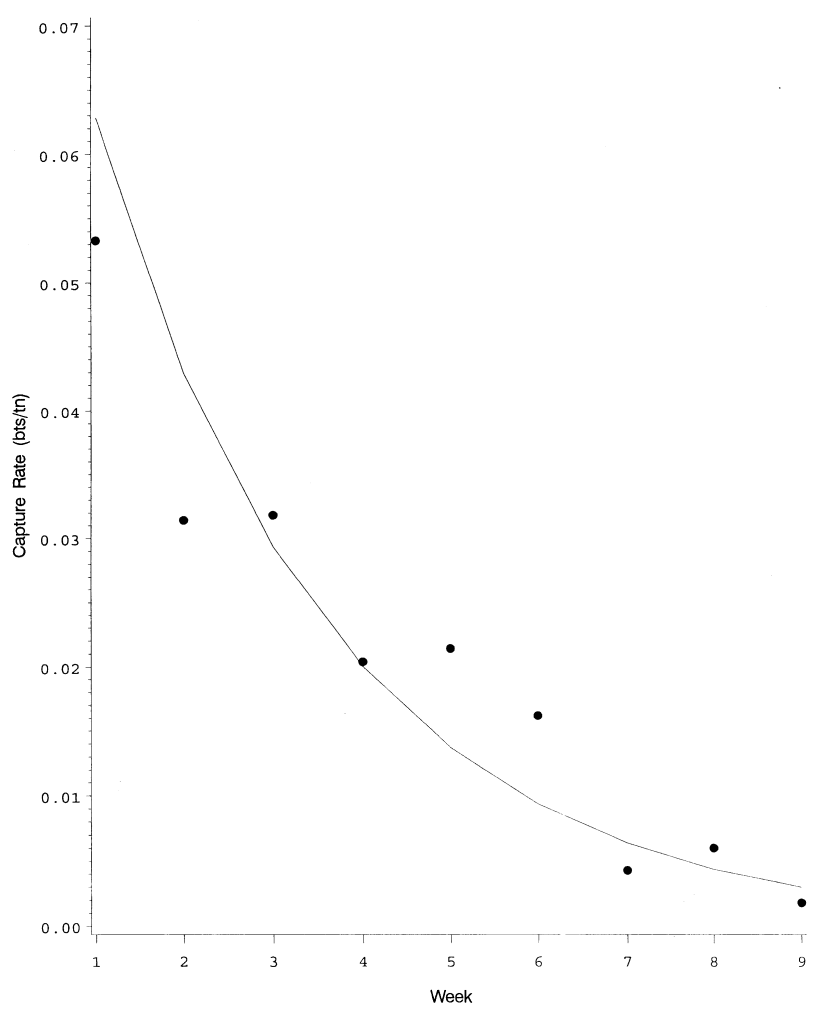

Fig. 1. Exponential decay model describing the brown tree snake capture rate (snakes/trap-night) by perimeter trapping from a 17.8 ha forested plot on Guam. $p=0.4618$ ). Rats (Rattus spp.) were the only nontarget animals captured in snake traps and they appeared with increasing frequency as trapping progressed through Phase 1 and 2 (Table 1).

\section{Discussion}

The rapid decline in capture rates to a low, relatively constant level has been demonstrated for other applications (Engeman and Linnell, 1998; Engeman et al., 1998c). That there were no time trends evident after the initial decline in Phase 1 and throughout Phase 2 suggests that the capture rate was in equilibrium with natural processes. This result, combined with very few captures in the interior and the fact that no difference was detected between the low-level steady state capture rates for Phase 1 and 2, leads us to conclude it is

Table 1

Capture summaries for two phases of trapping brown tree snakes from a 17.8 ha forested plot on Guam ${ }^{\mathrm{a}, \mathrm{b}}$

\begin{tabular}{|c|c|c|c|c|}
\hline Week & Date & Snake captures & Rate captures & Snakes/TN \\
\hline \multicolumn{5}{|c|}{ Phase 1: } \\
\hline 1 & $4 / 7-13$ & 8 & 0 & 0.0533 \\
\hline 2 & $4 / 14-20$ & 29 & 0 & 0.0314 \\
\hline 3 & $4 / 21-27$ & 31 & 0 & 0.0318 \\
\hline 4 & $4 / 28-5 / 4$ & 19 & 0 & 0.0204 \\
\hline 5 & $5 / 5-11$ & 25 & 0 & 0.0214 \\
\hline 6 & $5 / 12-18$ & 17 & 1 & 0.0162 \\
\hline 7 & $5 / 19-25$ & 5 & 0 & 0.0043 \\
\hline 8 & $5 / 26-6 / 1$ & 5 & 0 & 0.0060 \\
\hline 9 & $6 / 2-/ 8$ & 2 & 0 & 0.0018 \\
\hline 10 & $6 / 9-15$ & 7 & 0 & 0.0071 \\
\hline 11 & $6 / 16-22$ & 4 & 0 & 0.0043 \\
\hline 12 & $6 / 23-29$ & 5 & 0 & 0.0047 \\
\hline 13 & $6 / 30-7 / 6$ & 4 & 0 & 0.0039 \\
\hline 14 & $7 / 7-13$ & 4 & 0 & 0.0031 \\
\hline 15 & $7 / 14-20$ & 3 & 0 & 0.0037 \\
\hline 16 & $7 / 21-27$ & 6 & 0 & 0.0048 \\
\hline 17 & $7 / 28-8 / 3$ & 3 & 0 & 0.0048 \\
\hline 18 & $8 / 4-10$ & 8 & 0 & 0.0053 \\
\hline 19 & $8 / 11-17$ & 7 & 0 & 0.0054 \\
\hline 20 & $8 / 18-24$ & 8 & 1 & 0.0074 \\
\hline 21 & $8 / 25-31$ & 3 & 1 & 0.0026 \\
\hline 22 & $9 / 1-7$ & 4 & 1 & 0.0037 \\
\hline \multicolumn{5}{|c|}{ Phase 2: } \\
\hline 23 & $9 / 8-14$ & 8 & 2 & 0.0078 \\
\hline 24 & $9 / 15-21$ & 10 & 0 & 0.0095 \\
\hline 25 & $9 / 22-28$ & $8^{\mathrm{c}}$ & 3 & 0.0046 \\
\hline 26 & $9 / 29-10 / 5$ & 10 & 0 & 0.0095 \\
\hline 27 & $10 / 6-12$ & 4 & 0 & 0.0038 \\
\hline 28 & $10 / 13-19$ & 1 & 0 & 0.0010 \\
\hline 29 & $10 / 20-26$ & 7 & 4 & 0.0037 \\
\hline 30 & $10 / 27-31$ & 7 & 0 & 0.0050 \\
\hline
\end{tabular}

\footnotetext{
${ }^{\text {a }}$ Phase 1 placed traps only on the plot perimeter.

${ }^{b}$ Phase 2 had traps placed on the perimeter and interior of the for-

${ }^{\mathrm{c}}$ Five of these eight snakes were taken in interior traps and were the only snakes captured in interior traps.
} est. 
highly unlikely that a significant untrapped reservoir of brown tree snakes remained in the plot interior after the plot had been trapped on its perimeter. This is supported by results from smaller plots where trapping essentially removed the brown tree snake population without leaving behind a substantial untrappable population (Engeman et al., 1998a). The low level capture rate of brown tree snakes that remained from week 7 of Phase 1 for the following 23 weeks till the completion of the study likely represent recruitment to the plot population, probably mostly through re-invasion and, to a lesser degree in this time frame, from maturation of resident adolescent snakes to a trappable size. The increasing appearance of rats as nontarget captures served as an additional, but indirect, indication of a decrease in their primary predator, brown tree snakes.

Some important management implications obtain from the results of this study. First, although this 7 month study monitored a single large plot, the results paralleled those from many other plots of varying sizes from 3.6 to $8.4 \mathrm{ha}$. This study demonstrated that brown tree snake populations can be substantially reduced in a nearly 18 ha plot through perimeter trapping, similar to the reductions observed for much smaller plots. Trapping larger plots only on the perimeter presents a savings in labor because plots of this size no longer need to be subdivided by cutting trails through them for trap lines, and trapping only on the perimeter allows for vehicle access to maintain traps. Personnel and trapping materials can be extended further to cover more and larger plots only on the perimeter. This could be especially valuable when preparing larger areas of land for reintroduction of native species.

Also, a number of chemically contaminated sites can be found adjacent to some port areas on Guam. They have been considered too hazardous to trap on their interiors, but their proximity to cargo creates a risk for their snake populations to enter the transportation flow from Guam. Perimeter trapping can now be applied with greater confidence that the snake populations throughout the plot will be removed.

Fitting an exponential decay model to describe the decline in capture rates also has implications for management (Engeman and Linnell, 1998). The supply of traps available for control work and the personnel required to tend them are limited resources. Efficient long-term planning for the use of control resources would be benefitted if the removal rate of brown tree snakes could be predicted. As data from multiple plots are examined for their fit to the same functional model, a general random coefficient model (Laird and Ware, 1982; Littell et al., 1996) might be developed to describe snake removal from a generic plot, giving managers predictive capability.

\section{Acknowledgements}

We thank J. Brooks, M. Fall, T. Fritts for reviews of this manuscript. J. Bourassa provided valuable assistance with measuring the area of the plot using GIS analyses.

\section{References}

Engeman, R.M., Linnell, M.A., 1998. Trapping strategies for deterring the spread of brown tree snakes (Boiga irregularis) from Guam. Pacific Conservation Biology 4, 348-353.

Engeman, R.M., Linnell, M.A., Pochop, P.A., Gamboa, J., 1998 a. Substantial reductions of brown tree snake (Boiga irregularis) populations in blocks of land on Guam through operational trapping. International Biodegradation and Biodeterioration 46, 167171.

Engeman, R.M., Linnell, M.A., Vice, D.S., Pitzler, M.E., 1998 b. Efficacy of the methods used in an integrated program to deter the spread of brown tree snakes from Guam. Proceedings Australian Vertebrate Pest Conference 11, 435-440.

Engeman, R.M., Sayama, S., Linnell, M.A., 1998c. Operational utility of perimeter trapping for removing brown tree snakes (Boiga irregularis) from a defined area. The Snake 28, 19-22.

Fritts, T.H., McCoid, M.J., 1991. Predation by the brown tree snake (Boiga irregularis) on poultry and other domesticated animals on Guam. The Snake 23, 75-80.

Fritts, T.H., McCoid, M.J., Haddock, R.L., 1990. Risks to infants on Guam from bites of the brown tree snake (Boiga irregularis). American Journal of Tropical Medicine and Hygiene 42, 607611.

Fritts, T.H., Scott, N.J., Savidge, J.A., 1987. Activity of the arboreal brown tree snake (Boiga irregularis) on Guam as determined by electrical outages. The Snake 19, 51-58.

Laird, N.M., Ware, J.H., 1982. Random-effects models for longitudinal data. Biometrics 38, 963-974.

Linnell, M.A., Engeman, R.M., Pitzler, M.E., Watten, M.O., Whitehead, G.F., Miller, R.C., 1998. An evaluation of two designs of stamped metal trap flaps for use in the operational control of brown tree snakes (Boiga irregularis). The Snake 28, 14-18.

Linnell, M.A., Rodriguez, D.V., Mauldin, R.E., Engeman, R.M., 1997. Boiga irregularis: incubation and diet. SSAR Herpetological Review 28, 153.

Littell, R.C., Milliken, G.A., Stroup, W.W., Wolfinger, R.D., 1996. SAS System for Mixed Models. SAS Institute, Cary, NC, p. 633.

McCoid, M.J., Fritts, T.H., Campbell, E.W., 1994. A brown tree snake (Colubridae: Boiga irregularis) sighting in Texas. Texas Journal of Science 46, 365-368.

National Research Council, 1997. The Scientific Bases for the Preservation of the Mariana Crow. National Academy Press, Washington, DC.

Rodda, G.H., Fritts, T.H., 1992. The impact of the introduction of the colubrid snake Boiga irregularis on Guam's lizards. Journal of Herpetology 26, 166-174.

Rodda, G.H., Fritts, T.H., Chiszar, D., 1997. The disappearance of Guam's Wildlife. Bioscience 47, 565-574.

Rodda, G.H., Fritts, T.H., Conry, P.J., 1992. Origin and population growth of the brown tree snake, Boiga irregularis, on Guam. Pacific Science 46, 46-57.

Savidge, J.A., 1987. Extinction of an island forest avifauna by an introduced snake. Ecology 68, 660-668.

Wiles, G.J., Aguon, C.F., Davis, G.W., Grout, D.J., 1995. The status and distribution of endangered animals and plants in northern Guam. Micronesica 28, 31-49. 